\title{
Evaluation of residual stresses in laser clad coating at the micrometer scale
}

\author{
I. Furár, V. Ocelík \& J. Th. M. De Hosson \\ Materials innovation institute M2i, Department of Applied Physics, \\ University of Groningen, The Netherlands
}

\begin{abstract}
Residual stresses may have beneficial or detrimental effects to the materials properties and therefore precise knowledge and control of residual stresses are of high practical relevance. For the stress-relaxation measurement at a micro level we explored the slit milling (crack compliance) method using Focused Ion Beam (FIB) to release local strain induced by internal stresses and Digital Image Correlation (DIC) of SEM images taken before and after stress release to register it. The method allows us to measure stress components through the entire area of laser clad coating as a function of laser clad depth. We used: (i) $3.3 \mathrm{~kW}$ IPG fiber laser for cladding of tool steel, (ii) LYRA Tescan dual beam system with Focused Ion Beam and Electron Beam guns like milling-imaging instrument, respectively, (iii) Aramis GOM GmbH Digital Image Correlation for surface displacements registration. Finally, reducing the noise by using the appropriate magnification, facets size, resolution and by applying of integration method during collecting of SEM images we were able to increase sensitivity for lowest detectable displacement $(\sim 1 \mathrm{~nm})$.
\end{abstract}

Keywords: residual stress, focus Ion beam, laser clad coating, digital image correlation.

\section{Introduction}

Laser cladding is an important component of surface processing technology. Due to the small melting pool, large temperature gradient, narrow bonding zone and high thermal stress, cracks can be easily nucleated, especially in thick metallic coatings $[1,2]$. To prevent cracking is one of the most important issues from the preparation and industrial points of view [3-5]. Therefore an evaluation of 
residual stresses in laser clad coating at a micrometer scale provides useful information about the whole profile of internal stresses inside laser clad coating.

A number of methods have been developed for quantifying residual stresses. Most of these methods determine the mean stresses in relatively large volumes. They give suitable information when residual stresses are distributed homogeneously and stress gradients are absent [6]. However, in many cases the residual stresses are not distributed homogeneously over the investigated volume. Therefore in many applications in engineering a number of nondestructive techniques, such as X-ray, neutron diffraction, moiré interferometry [7-11] and destructive and semi-destructive methods like layer removal and curvature measurement, incremental blind hole drilling, crack compliance, cantilever method etc. [12-19] have been developed, based on material removal and the measurement of the associated strain relief. Figure 1 compares a number of methods with respect to their sensitivity and length scale.

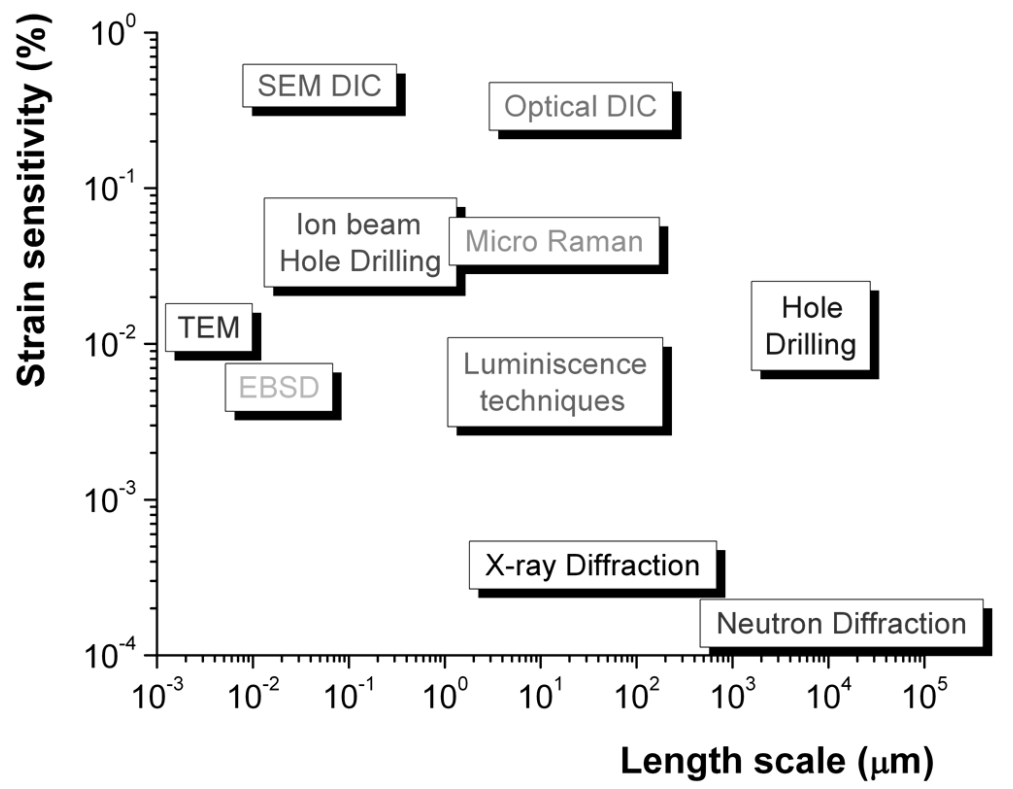

Figure 1: An overview of different strain measurement methods [20].

In this work a relatively new approach of stress measurement on the micron scale, based on applying dual beam microscopy like imaging-milling and semidestructive instrument to realize a mechanical relaxation method of measuring internal stress was used. Focused Ion Beam (FIB) was used as a "sharp knife" to release local strain induced by internal stresses. Surface relaxation in the vicinity of such a cut was mapped using the Digital Image Correlation (DIC) and released stresses were calculated on the base of these displacement maps. One of the goals of this work is to prove that this microscopic method of measuring internal stresses is appropriate for laser clad coatings. 


\section{Experimental}

\subsection{Sample preparation}

The sample used for measuring internal stress was cut longitudinally to the cladding direction from $8 \mathrm{~mm}$ bearing steel bar clad with $1 \mathrm{~mm}$ thick MicroMelt23 coating. Longitudinal cut (shown in Fig. 2a) does not release the main stress inside cladding layer. Surface for measurement was subjected to the standard metallographic procedure starting with grinding on grit papers (120, $320,800,1200$ and 4000), and finishing with $\sim 1 \mathrm{~h}$ polishing using the Struers OP-U suspension. The spherical nano sized $(25 \pm 5 \mathrm{~nm})$ Yttrium-stabilized Zirconia (YSZ) particles with appropriate distribution and density were used to enhance the contrast of SEM surface images. These particles precipitated on the surface of the specimen shown in Fig. $2 \mathrm{~b}$ from the ethanol suspension which was in an ultrasonic bath for 30 minutes to brake-up the large particle conglomerates $[16,17]$.
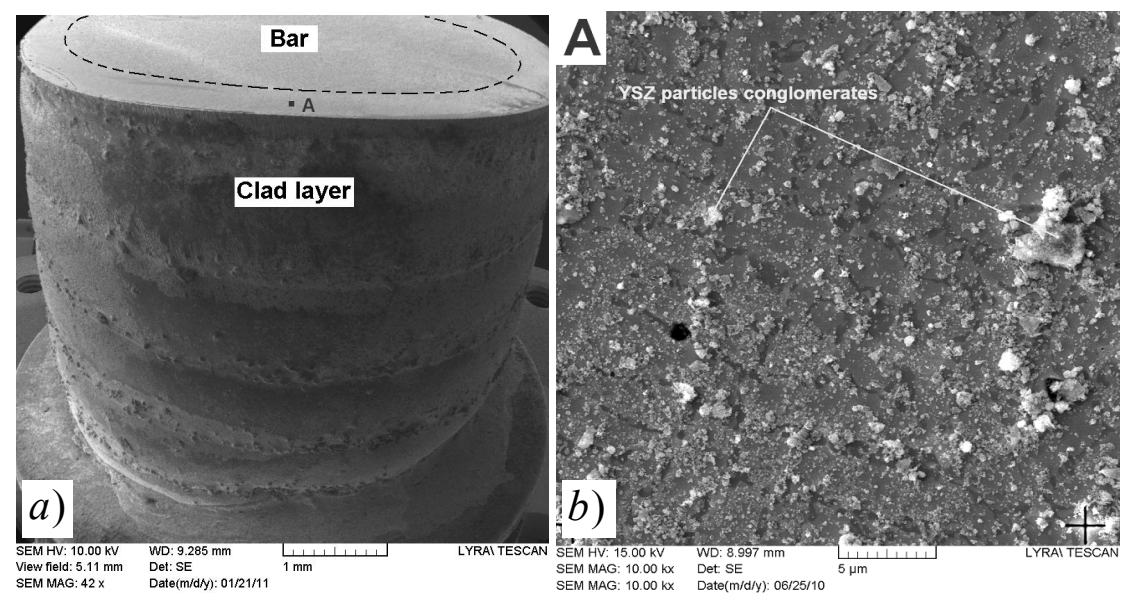

Figure 2: $\quad$ SEM image of laser clad sample cut for internal stress measurements (a) and a detail of the measured surface area with YSZ particles (b).

\section{Results and discussion}

\subsection{FIB milling}

For the stress-relaxation we used the idea of the macroscopic crack compliance method [15]. The Focused Ion Beam (FIB) mills a slit with appropriate parameters; like $2.8 \mu \mathrm{m}$ in depth, $25 \mu \mathrm{m}$ in length and $0.5 \mu \mathrm{m}$ in width using the focused $\mathrm{Ga}^{+}$ion beam of $200 \mathrm{pA}$ and accelerating voltage of $30 \mathrm{kV}$.

Each picture was taken with the electron beam perpendicular to the surface with the image resolution of $768 \times 768$ pixels and magnification of $5 \mathrm{k}$. Electron 
beam was accelerated by an electric field of $10 \mathrm{kV}$. The SEM image was collected using the dwell time $t_{D}=48.6 \mu \mathrm{s} /$ pixel.

\subsection{DIC analysis}

Aramis GOM GmbH, a complex deformation analysis system was used for measuring the displacements initiated by internal stress release. DIC is a computational method to calculate displacements field by correlation of small facets between two digital images, the first one taken before (reference one) and the second one after the deformation. Typical standard deviation of the displacement error for DIC algorithm is reported as 0.01 pixels [21]. DIC calculates a discrete displacement vector $\left(D_{x}, D_{y}\right)$ in the centers of rectangular facets. These centers represent a rectangular grid at the measured surface with a size for instance $21 \times 21$ pixels that uniformly covers the area around the slit. Figure $3 \mathrm{a}$ shows this calculated area that almost fully covers the whole SEM image. Brightness intensity corresponds to the measured displacement value according scale on the right side. For a better visualisation of the displacement area around the slit the displacement scale has been changed from $\pm 50 \mathrm{~nm}$ to $\pm 25 \mathrm{~nm}$.
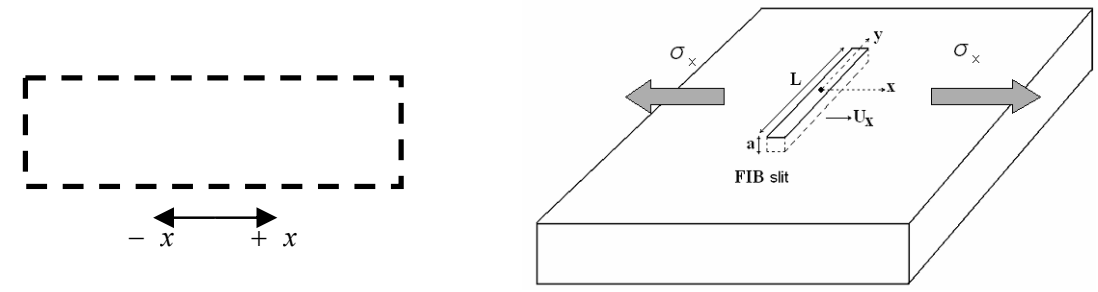

Figure 3: Left: SEM image of a region after the introduction of the FIB slit with the measured displacement area; right: a schematic picture of FIB slit with defined coordinates.

\subsection{Residual stress calculation}

To determine the residual stress from the measured displacements we used Inglis-Muskhelishvili solution for displacement in $x$ direction $u_{x}$ in an infinitely thick plate and for an infinitely long crack [22]:

$$
u_{x}=\frac{2.243}{E^{\prime}} \sigma_{x} \int_{0}^{a} \cos \theta\left(1+\frac{\sin ^{2} \theta}{2(1-v)}\right) \cdot(1.12+0.18 \cdot \sec h(\tan \theta)) d a,
$$

where $E^{\prime}=E /\left(1-v^{2}\right), E$ being Young's modulus, $v$ the Poisson's ratio. $\sigma_{x}$ is the residual stress in $\mathrm{x}$-direction, $\theta=\arctan (x / a)$ where $a$ is the slit depth and $x$ is the distance from the slit. This calculation provides displacement at any distance from the slit in both directions. Figure 4 a shows the average displacements as a function of distance from the slit. The displacements where measured along horizontal lines within dashed area around the slit, which is not expected to be influenced by the ends of the slit. 
Due to the form of Eq. (1) it is possible to estimate the size of internal stress $\sigma_{x}$ by plotting of observed displacements $u_{x}$ against calculated ones, assuming an internal stress (e.g. $\sigma_{x D}=-1 \mathrm{GPa}$ ). This plot is shown in Fig. $4 \mathrm{~b}$ together with a linear fit. The slope of this fit corresponds to the value of real internal stress released from the sample during formation of the slit [23]. As Fig. $4 \mathrm{~b}$ shows, the compressive residual stress of $-960 \pm 14 \mathrm{MPa}$ has been released by the introduction of a vertical slit. Because this slit was made perpendicular to the cladding direction the detected internal stress is oriented in the main cladding direction.
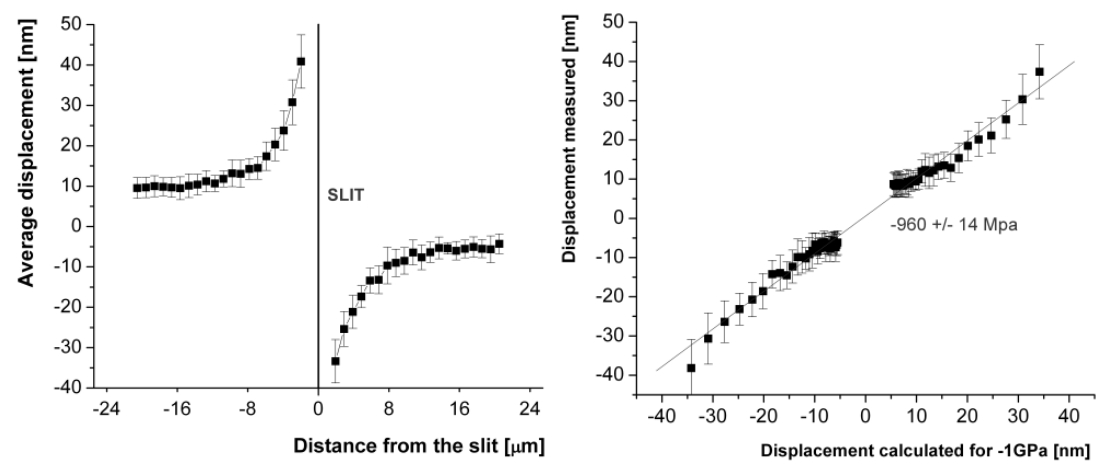

Figure 4: Left: The average of horizontal displacement on both sides of the slit as a function of distance from the slit; right: the measured averaged displacements plotted versus displacement calculated assuming a stress $(-1 \mathrm{GPa})$ with the linear fit.

\subsection{Displacement noise and stability tests}

As regards the sensitivity it is very interesting to study the lowest displacements that could be registered by DIC of SEM images. We defined the stability of SEM images as DIC noise between two images taken at the same conditions from the same place with a time delay between them. If there is no noise contribution from the SEM machine, then noise on the level of 0.01 pixel could be expected as a characteristic noise for DIC algorithm [21].

To test this stability we used the following experimental conditions: magnifications of $7 \mathrm{k}, 10 \mathrm{k}$ and $20 \mathrm{k}, 180$ seconds of time delay between 3 subsequent images, image resolutions of $768 \times 768$ and $1024 \times 1024$ pixels, dwell time $t_{D}=48.6 \mu \mathrm{s} /$ pixel, facet sizes of $43 \times 43$ and $21 \times 21$ pixels with a step size between them of 21 and 5 pixels, respectively. To simulate the FIB milling procedure the sample stage was moved and returned back into the same position, between taking the images. Figure 5 demonstrates the fields of horizontal (Fig. 5a) as well as vertical (Fig. 5b) displacement measured by DIC algorithm (facet size $43 \times 43$ pixels) between two subsequent SEM images from the same place taken at magnification $7 \mathrm{k}$ with the resolution of $768 \times 768$ pixels. The 
average displacements in both directions define the movement of the second image towards the first one, due to the stage manipulation. However, the variations of these displacements $(2.4 \mathrm{~nm}$ in $\mathrm{x}$ and $3.9 \mathrm{~nm}$ in y direction) characterize the average noise introduced by both: SEM column and DIC algorithm sources. This noise corresponds to the value that about 5 time larger than basic DIC noise (0.01 pixel).
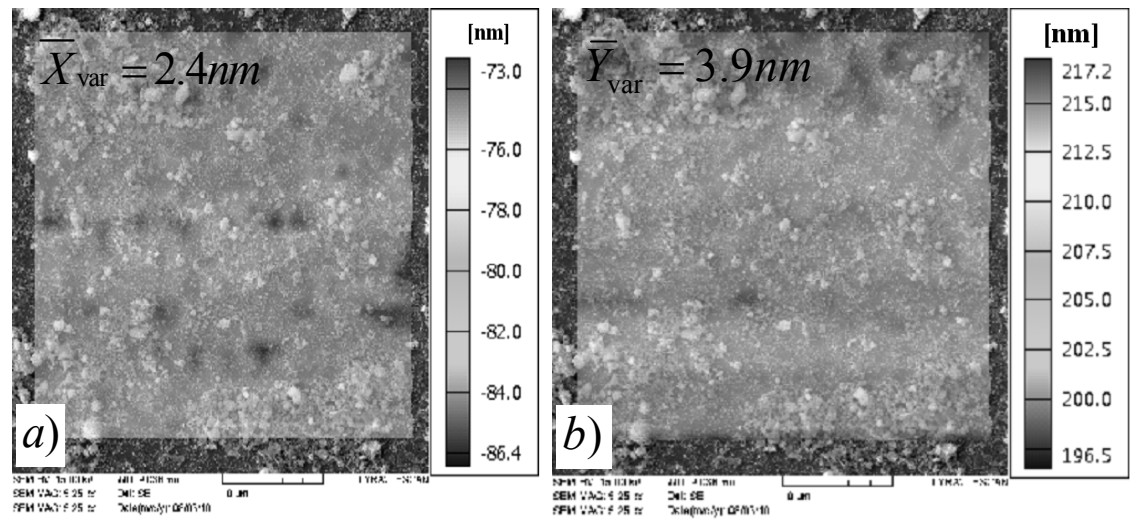

Figure 5: Displacements in X-horizontal (a) and Y-vertical (b) directions with magnification $7 \mathrm{k}$, image resolution $768 \times 768$, facet size $43 \times 43$, $53.6 \mathrm{~nm} / \mathrm{pixel}, t_{D}=48.6 \mu \mathrm{s} / \mathrm{pixel}$ facet step 21 pixels, calculated by DIC with the reference image.

Figure 6 summarizes our average noise measurements for different magnifications, facet sizes and image resolutions for both: vertical and horizontal directions. This figure clearly demonstrates that the images with resolution $768 \times 768$ shown generally lower noise than the images with higher resolution 1024x1024. From point of view of magnification and facet size, the magnification $10 \mathrm{k}$ and facet size $43 \mathrm{x} 43$ have shown the lowest noise introduced by SEM column.

A closer observation of Fig. 5a), b) reveals the character of the noise introduced by SEM column. This noise does not have a random character. On the contrary, the areas of similar values of displacements are grouped in the horizontal bands with different thicknesses. It is clear that these bands originate from the way how the SEM image is collected $[24,25]$. The electron beam scans the sample surface in horizontal lines from top to the bottom of the image. Each line is scanned from left to the right making a required amount of stops (768 or 1024) at equidistant positions using a preset dwell time. The displacements are grouped in bands with a width of about 75 pixels. 

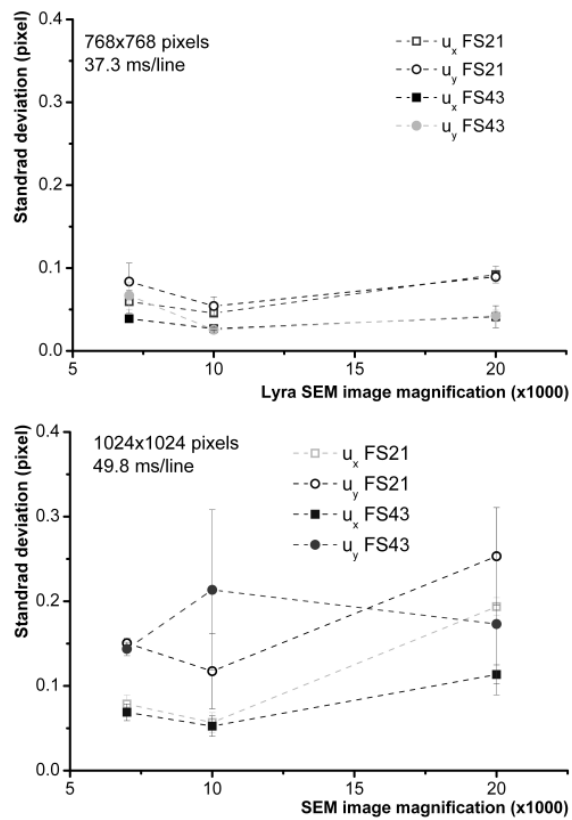

Figure 6: Standard deviation in $\mathrm{X}$ and $\mathrm{Y}$ displacements averaged in three subsequent stages for three different SEM image magnifications, two SEM image resolutions and two different facet sizes.

This corresponds to the time of about 3 seconds (the time in which the beam quickly moves from end of the line to the beginning of the next one has to be included). It is clear that time interval in which the microscope is relatively stable is about this time.

This leads us to the idea to collect images in a different regime, i.e. to integrate $\mathrm{m}$ SEM images taken with a much lower dwell time (the total scanning time has to be smaller than $\sim 3 \mathrm{~s}$ ) in such a way, that the total dwell time will be comparable with a dwell time used in slow scanning mode. In this way we should record an image with similar noise to signal ratio but less distorted by SEM column instability. The results of this test are shown in Fig. 7. Both, horizontal and vertical displacements are shown for slow scanning mode (Fig. 7a),b)) and integrated fast scanning mode (Fig. 7c),d)) when each SEM image was averaged from 30 scans with the dwell time of $t_{D}=1.8 \mu \mathrm{s} /$ pixel. It is clearly visible, than not only the ribbon structure of SEM column noise disappeared but moreover that the value of noise variation has been decreased. From these results it is clear that for SEM image magnification $7.5 \mathrm{k}$ and for image resolution of $768 \times 768$ the smallest displacements possible to register by DIC of SEM images are on the level of $1 \mathrm{~nm}$. 

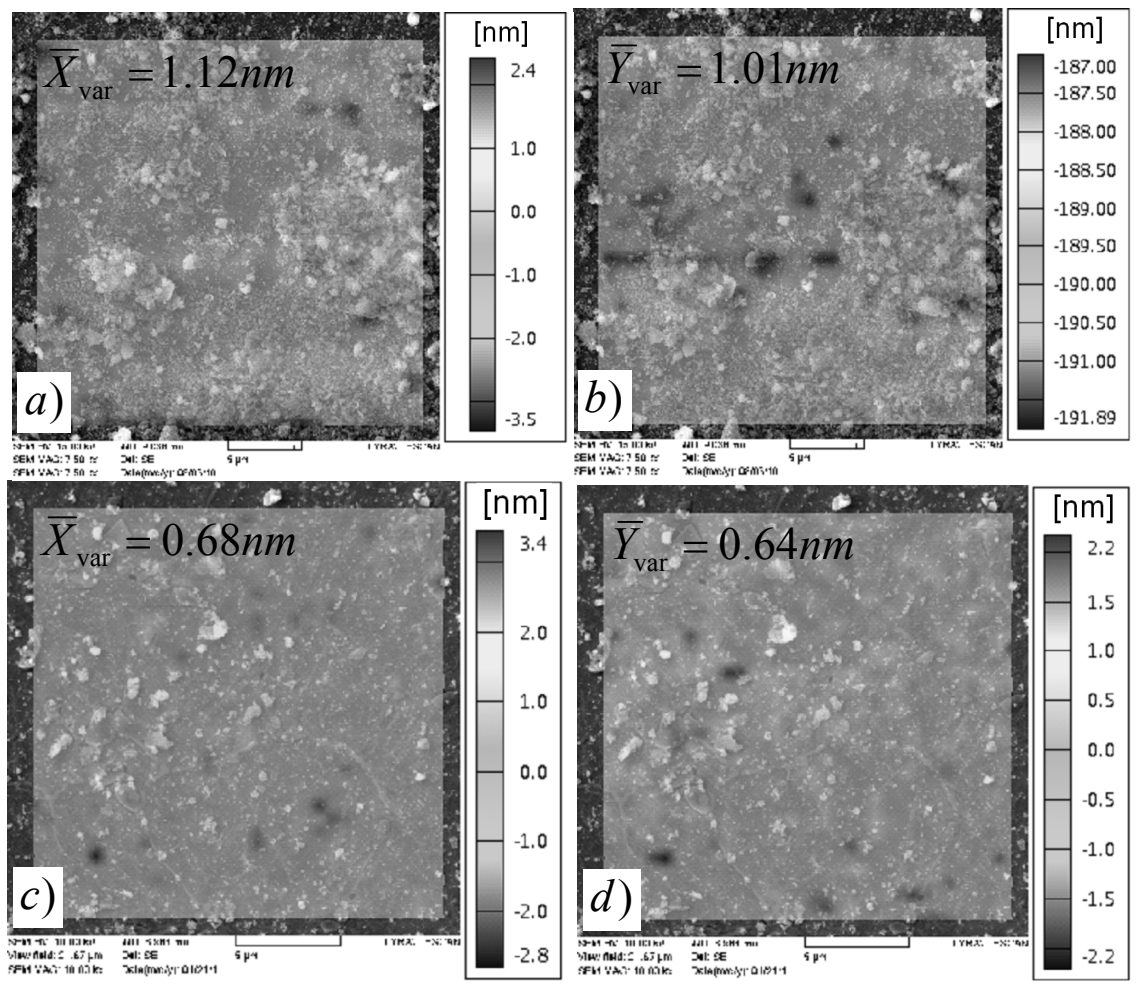

Figure 7: DIC on two standard image acquisition with magnification $10 \mathrm{kx}$, image resolution $768 \times 768,28.18 \mathrm{~nm} / \mathrm{pixel}, t_{D}=48.6 \mu \mathrm{s} / \mathrm{pixel}$ of displacement in X-horizontal (a) and Y-vertical (b) direction and integration method of image.

\section{Conclusions}

In summary, we explored the slit milling (crack compliance) method using the Focused Ion Beam to release the local strain induced by internal stresses and the Digital Image Correlation of scanning electron microscopy images taken before and after stress register this strain. The method allows measuring of stress components through the whole area of the laser clad coating as a function of depth.

Reducing the noise by using the appropriate magnification, facets size, picture resolution and by applying of integration method during collecting of SEM images we were able to increase sensitivity for lowest detectable displacement $(\sim 1 \mathrm{~nm})$.

From a practical point of view this method is universal and applicable for different types of materials like metals (crystalline and amorphous), ceramics, plastics and for different coatings like laser clad, PVD and spin coatings. 


\section{Acknowledgement}

The work is part of the research program of M2i (Materials innovation institute, The Netherlands) Project number: MC7.06259.

\section{References}

[1] Ocelík V, De Hosson JTM. Thick metallic coatings by coaxial and side laser cladding: processing and properties. In: Advances in Laser Materials Processing. Eds. Lawrence, J. Pou, D. K. Y. Low and E. Toyserkani, Woodhead Publishing Limited and CRC Press, Oxford 2010, Chapter 15, p.426-458.

[2] De Hosson JTM, Ocelík V, De Oliveira UOB, Vainchtein DI. International journal of materials research 2009;100:1343-1360.

[3] Zhou S, Zeng X, Hu Q, Huang Y. Applied surface science 2008;255:16461653.

[4] Wang D-s, Liang E-j, Chao M-j, Yuan B. Surface and coatings technology 2008;202:1371-1378.

[5] Wang F, Mao H, Zhang D, Zhao X, Shen Y. Applied surface science 2008;255:3267-3275.

[6] Massl S, Keckes J, Pippan R. Acta Materialia 2007;55:4835-4844.

[7] Nobre JP, Kornmeier M, Dias AM, Scholtes B. Experimental mechanics 2000;40:289-297.

[8] Meixner M, Fitzpatrick ME, Reimers W. Composites science and technology 2011;71:167-176.

[9] Dubois JB, Thilly L, Renault PO, Lecouturier F, Di Michiel M. Acta materialia 2010;58:6504-6512.

[10] Yi J, Bin-shi X, Hai-dou W, Ming L, Yao-hui L. Applied surface science 2011;257:2332-2336.

[11] Han B, Post D, Ifju P. Journal of strain analysis for engineering design 2001;36:101-117.

[12] Korsunsky AM, Sebastiani M, Bemporad E. Materials Letters 2009;63:1961-1963.

[13] Schajer GS. Experimental mechanics 2010;50:1117-1127.

[14] Sabaté N, Vogel D, Keller J, Gollhardt A, Marcos J, Gràcia I, Cané C, Michel B. Microelectronic engineering 2007;84:1783-1787.

[15] Cheng W, Finnie I. Engineering fracture mechanics 1993;46:79-91.

[16] Winiarski B, Langford RM, Tian J, Yokoyama Y, Liaw PK, Withers PJ. Metallurgical and materials transactions a: physical metallurgy and materials science 2010;41:1743-1751.

[17] Winiarski B, Withers PJ. Mapping Residual Stress Profiles at the Micron Scale Using FIB Micro-hole Drilling, vol. 24-25, 2010.

[18] Wang Q, Ishikawa H, Nakano S, Ogiso H, Akedo J. Vacuum 2004;75:225229.

[19] Massl S, Keckes J, Pippan R. Scripta Materialia 2008;59:503-506. 
[20] Dingley DJ, Wilkinson AJ, Meaden G, Karamched PS. Journal of electron microscopy 2010;59:S155-S163.

[21] Shen B, Paulino GH. Exp Mech 2010;51:143-163.

[22] Tada H., Paris P., Irwin G. In: The Stress Analysis of Cracks. UK: Bury St. Edmunds, 2000, pp. 82-96.

[23] Kang KJ, Yao N, He MY, Evans AG. Thin solid films 2003;443:71-77.

[24] Sutton MA, Li N, Garcia D, Cornille N, Orteu JJ, McNeill SR, Schreier HW, Li X, Reynolds AP. Exp mech 2007;47:789-804.

[25] Sutton MA, Li N, Joy DC, Reynolds AP, Li X. Exp mech 2007;47:775-787. 